\title{
Wenn Männer Milch geben
}

Ein 26-jähriger Mann berichtete, dass seit einem Jahr die Libido abgenommen habe und er unter einer erektilen Dysfunktion leiden würde. Er hatte keine Brustschmerzen, bemerkte aber einen weißlichen Ausfluss aus den Brustwarzen bei leichter Manipulation (Abb. 1).

- Kopfschmerzen, Sehstörungen und die Einnahme von Medikamenten oder Drogen wurden verneint. Bei der Untersuchung zeigte sich eine beidseitige Gynäkomastie und eine auffallend geringe Gesichts- und Körperbehaarung. Das Hodenvolumen betrug beidseits $10 \mathrm{ml}$ (Normal 12-12 ml). Bei der Untersuchung des Gesichtsfeldes zeigte sich eine bitemporale Hemianopsie. Es bestand eine Euthyreose. Die hypophysären Hormone wie LH, FSH, ACTH und Wachstumshormon lagen im Normbereich. Auffallend waren ein Prolaktinspiegel von $13050 \mathrm{pmol} / \mathrm{l}$

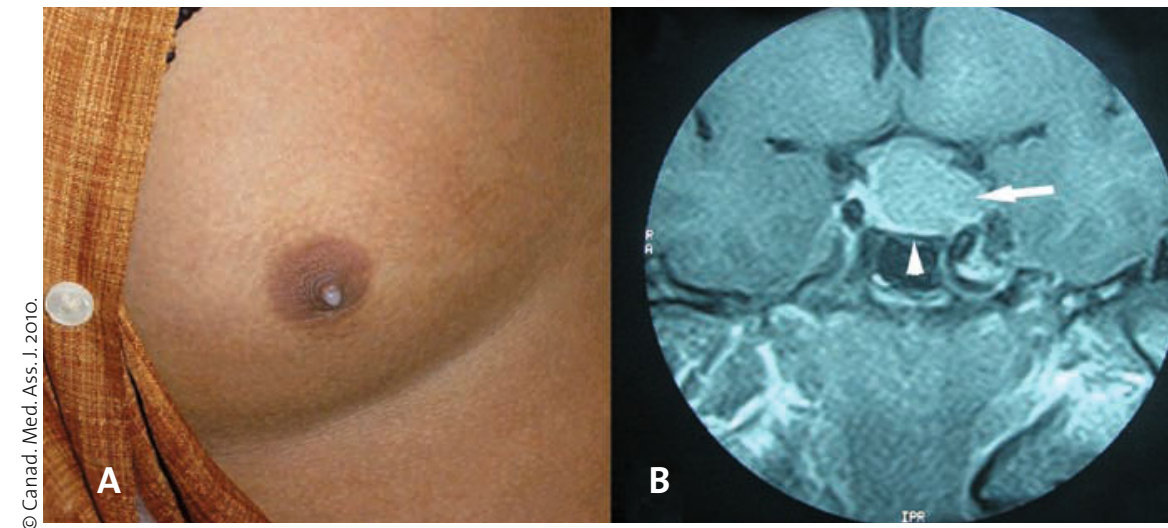

Ausfluss aus der Brustwarze (A) und Kernspintomografie der Hypophyse (B).

(Normal 109-522 pmol/l) und ein Serumtestosteron von nur $6,4 \mathrm{nmol} / 1$ (Normal 10,4-31,0 nmol/l).

In der Kernspintomografie stellte sich ein 2,2 x 1,8 x 1,2 cm großer Hypophysentumor dar. Nach sechs Monaten Behandlung mit Bromocriptin hatte das Serumprolaktin nahezu den oberen
Normbereich erreicht, war das Testosteron auf $12,0 \mathrm{nmol} / \mathrm{l}$ angestiegen und hatte sich die klinische Symptomatik wie Libido und sexuelle Aktivität normalisiert. Eine kernspinomografische Kontrolle der Hypophyse nach einem halben Jahr zeigte eine deutliche Größenabnahme des Tumors.

\section{Kommentar}

Eine Galaktorrhö tritt bei etwa $80 \%$ der Frauen mit Prolaktinom auf, ist jedoch bei Männern sehr ungewöhnlich. Die Symptomatik von Prolaktinomen bei Frauen bezieht sich in der Regel auf eine Amenorrhö und Galaktorrhö, die bereits bei Mikroadenomen von weniger als $1 \mathrm{~cm}$ Größe auftreten kann. Bei Männern sind die entdeckten Prolaktinome in aller Regel größer und verursachen dadurch auch klinische Symptome aufgrund von Verdrängungseffekten. Die meisten Prolaktinome können konservativ nur mit Dopaminagonisten gut behandelt werden.

Die Empfehlungen zur Überwachung beinhalten eine jährliche Messung des Serumprolaktins und eine klinische Untersuchung, wobei besonders auf Kopfschmerzen und Gesichtsfeldausfälle zu achten ist. Eine Wiederholung der Kernspintomografie der Hypophyse wird empfohlen bei Prolaktinspiegeln über 10000 pmol/l. Bei Patienten mit Makroadenomen sollte die Kernspintomografie routinemäßig alle zwei bis drei Jahre wiederholt werden.

Eine chirurgische Therapie ist den Patienten vorbehalten, die auf Dopaminagonisten schlecht ansprechen, oder bei denen Nebenwirkungen vorherrschen. Bei etwa $5 \%$ der Patienten wird eine tatsächliche Resistenz gegen Dopaminagonisten beobachtet. 5-10\% tolerieren die Therapie nicht wegen orthostatischer Beschwerden, Übelkeit bis hin zum Erbrechen und anhaltendem Schwindel.

Diese Nebenwirkungen treten in geringerem Umfang zu Beginn der Behandlung auf. Daher sollte die dopaminagonistische Therapie immer einschleichend begonnen und die Dosis möglichst in den Abendstunden verabreicht werden. Wie lange diese Therapie fortgeführt werden muss, ist noch nicht schlüssig bekannt. In retrospektiven Studien bei Patienten mit Makroprolaktinomen kam es nach dem Absetzen mit wenigen Ausnahmen immer zu einem Wiederanstieg des Prolaktins und zu einer Größenzunahme des Hypophysenadenoms. In neueren prospektiven Studien blieb nach einer mindestens zweijährigen Therapie mit Cabergolin der Prolaktinspiegel bei immerhin 36\% der Patienten im Normbereich. Wahrscheinlich ist also doch eine lebenslange Therapie erforderlich.

H. S. FüEßL =
- T. M. Anoop et al.

(Korr.: T. M. Anoop, MD, Department of Medicine, Kottayam Medical College, Kerala, India). Lactation associated with a pituitary tumour in a man. Canad. Med. Ass. J. 2010. DOI:10.1503/cmaj.ogo888. 